\title{
Strategies and Insights on Digital Inclusion of Older People in Hong Kong
}

\author{
Famin Wang ${ }^{1}$, Runxuan Zou ${ }^{1, *}$, Jing Tang ${ }^{2}$
}

\begin{abstract}
${ }^{1}$ School of Marxism, South China Normal University, Guangzhou, Guangdong, China
${ }^{2}$ School of history and culture, South China Normal University, Guangzhou, Guangdong, China

*Corresponding author. Email: 591631958@qq.com
\end{abstract}

\begin{abstract}
To address the digital divide among older adults in Mainland China, this study examines Hong Kong's initiatives to promote digital inclusion of older adults based on a literature analysis approach. The study finds that Hong Kong has been effective in bridging the digital divide among the elderly by improving their digital access and encouraging them to use information technology. In Mainland China, the digital divide among the elderly can be addressed by strengthening the government's leadership and comprehensive promotion, safeguarding the "digital rights" of the elderly, continuously improving the digital literacy of the elderly, and promoting the digital inclusion of the elderly and the young.
\end{abstract}

Keywords: Aging education, Lifelong learning, Digital inclusion, Digital divide.

\section{香港老年群体数字融入的策略与启示}

\section{王发民 ${ }^{1}$, 邹润轩 ${ }^{1, *}$, 汤婧 ${ }^{2}$}

\author{
${ }^{1}$ 华南师范大学马克思主义学院, 广东广州, 中国 \\ 2 华南师范大学历史文化学院, 广东广州, 中国 \\ “通讯作者. 邮箱: 591631958@qq.com
}

\section{中文摘要}

为解决中国内地老年数字鸿沟问题, 本研究基于文献分析法对香港推进老年人数字融入的举措展开研究。研究 发现, 香港通过提升老年人的数字接入能力、鼓励老年人使用信息技术等方式来弥合老年人数字鸿沟, 收效良 好。中国内地可从加强政府引领全面推动、维护老年人 “数字权利” 、不断提升老年人数字素养和促进数字长 幼共融等维度来解决老年数字鸿沟问题。

关键词：老年教育，终身学习，数字融入，数字鸿沟

\section{1. 前言}

当今世界，人口老龄化和社会数字化并存。2018 年全球 65 岁及以上人口首次超过 5 岁以下人口数 量, 且增长速度最快 ${ }^{[1]}$, 同时数字化伴随信息技术及 其应用的快速发展已渗透到社会生活的各个方面。
这催生了数字鸿沟, 而老年数字鸿沟更为严峻。此 背景下, 加快老年人的数字融入成为重要课题。中 国香港在推进老年数字融入过程中进行了诸多探索 并取得了积极成效。因此，本文基于当前中国内地 老年人数字融入不足的挑战, 将探究香港促进老年 
人数字融入的举措及其经验, 旨在为中国内地老年 人融入数字生活提供启示。

\section{2. 中国内地老年人的数字融入困境及其原因 分析}

\section{1. 数字融入不足}

随着时代的发展，信息技术对老年人生活的影响 与日俱增。相关实证研究表明, 我国老年人的信息 能力与终身学习需求密切相关 ${ }^{[2]}$, 而且信息技术具有 激励老年人以新的方式积极参与生活的重要作用 ${ }^{[3]}$ 。 这意味着老年学习者要想与时俱进必须提升自身的 媒介与信息素养 (Media and Information Literacy, MIL), MIL 指的是一种 “能够批判地、合 理合法地获取、理解、评价、利用、创造和分享各 类型媒介信息与知识的综合能力（包含知识、技能 和态度）” ${ }^{[4]}$ 。然而, 当前中国内地老年人的 MIL 有 待提高, 致使他们不但难以获得数字红利, 而且也 常成为电信诈骗的主要受害对象, 截至 2020 年 12 月中国 60 岁及以上网民群体仅占网民总数 11.2\% 。

在世界范围内，老年人的信息技术能力普遍落后 于年轻人 ${ }^{[6][7]}$, 从而导致老年数字鸿沟问题, 进而使 得原本属于弱势群体的老人们更加被信息化社会边 缘化以致陷入不利处境, 沦为所谓的 “数字难 民” ${ }^{[8]}$ 。数字鸿沟是指在信息技术可及性以及使用方 面不同人之间存在的差异 ${ }^{[9]}$ 。黄晨喜指出, 老年人和 青年人在信息素质上差别极大, 无论在使用端、接 入端还是知识端 ${ }^{[10]}$ 。

总之, 数字鸿沟的存在导致老年人被排除在数字 化社会之外，无法平等享受包括信息获取、社会交 往、外卖、网购、网络教育等方面的数字红利, 进 而对他们的终身学习产生不利影响。因此, 在信息 社会, 中国内地老年人正面临数字融入不足的困境。

\section{2. 数字融入不足的原因分析}

当然，老年人数字融入不足是多种机制共同作用 的结果, 但主要有以下几个方面原因。一是个人原 因。生理上, 老年人的身体机能呈现下降的趋势, 视觉、听觉和反应速度等的日渐衰弱成为了他们使 用现代科技产品的障碍, 比如在操作电脑和智能手 机方面较之年轻人要困难很多。心理上, 随着学习 和认知能力的下降, 老年人面对科技产品时容易心 生无力感和恐惧感，对技术 “有用性” 和 “易用 性” 存在认知失调, 对社交媒体的积极作用理解不 足进而对其产生刻板印象, 加剧了老年人对社交媒 体技术的抗拒 ${ }^{[11]}$ 。此外, 受教育程度对老年人的数 字融入具有重要作用。二是家庭、社会和制度原因。 老人群体在信息科技方面的家庭反哺、亲友互助以 及社会支持不够, 特别是社会制度设计的不合理等 也是他们数字融入困难的重要原因 ${ }^{[12]}$ 。三是现代科 技本身的原因。现代科技产品具有 “老年歧视” 倾
向, 对老年人的数字需求缺乏靶向服务, 它们的设 计更多适用于年轻群体。面对纷繁复杂的科技产品, 老年群体更多的是手足无措和望 “云” 兴叹。

综上，个人、家庭、社会、制度和科技等诸多因 素共同导致了老年群体数字融入困难, 拉大了数字 鸿沟。这种因应时代发展的结构性变革致使老年群 体在与现代科技的互动过程中沦为弱势。

\section{3. 香港推进老年人数字融入的举措}

作为世界平均预期寿命最长和信息化发展水平 领先的地区, 香港同样面临老年人数字融入不足和 老年数字鸿沟的难题。为化解这一难题, 香港多年 来一直致力于探索解决方案, 并推出很多具有针对 性的举措。

\section{1. 营造环境，提升老年人数字接入能力}

针对老年人数字融入过程中面临的软件环境缺 失, 近年来香港社会各方积极为老年人提供靶向数 字应用软件环境。

一是建立老年群体专用网站。为满足老年人对福 利、保健和法律等与他们利益做关的信息的需求, 香港政府相关部门和非营利组织专门创设老年人网 站, 例如长青网、老友网和社会福利署长者资讯网 等。二是开发专用移动 App。2012 年, 香港相关部 门推出 “数码共融流动应用程式” 资助项目 ${ }^{[13]}$, 以 资助非营利组织开发老年人 APP, 并向老年人开放和 免费使用。此外，其他基金如 “科创生活基金” 等 也相继投入大量真金白银资助相关创新科技项目以 满足老年人等特殊群体的需要。三是定制无障碍软 件。针对老年人随着年纪增长而逐渐出现身体机能 和认知能力衰弱等问题, 资科办倡导相关机构比如 学校、企业等为老年人专门定制无障碍软件和网页 以及提供移动 App 专题讲座。

\section{2. 多措并举，提高老年人信息素养}

为提高老年人的信息素养，香港社会各界多措并 举向老年人提供多样化信息技术培训。

一是面授培训。首先, 香港各界基于老年人的需 求对他们进行面对面的信息技术培训，这也是他们 培训的主体。一些机构, 比如长者学苑, 利用自身 优势并结合老年人的需求开设相关课程对这些受众 展开面授培训, 以拉近老人与信息技术的距离, 从 而增进他们对数字化的了解, 进而提升他们应用信 息技术的能力。此外, 老年人还可以到那些向他们 开放的学校旁听相关信息技术课, 甚至可以修读专 业课程。其次，香港特区政府利用综合性项目和主 题培训类项目带动老年人的面授培训。此类项目通 过网站建设、软件比赛、巡回展览等方式为老年人 提供多样化的信息技术服务。总之，这类课程设计、 开发和实施规范，且极富时代感、针对性、吸引力 
和多元化，有时会邀请年轻学生和老年人互动并交 流数字生活经验, 促进长幼共融。

二是在线培训。为方便香港老年人在家学习信息 技术，相关部门为其提供网上培训课程，课程内容 包括网购、电子政务、网络安全、和社交媒体等。 这类课程灵活多样，授课老师可以实时与老年人进 行互动。

三是外展培训。这类培训主要针对老年人中的特 定群体。2014 年以来, 资科办陆续推出多轮 “长者 数码外展计划” [14]，旨在资助非营利机构对特定老 年人和患有认知障碍症老人进行移动设备培训, 以 提升其信息技术能力和兴趣。由于收效良好， 2018 年这类项目逐渐常态化。

\section{3. 多管齐下，鼓励老年人运用信息技术}

为鼓励老年人身体力行使用信息技术，香港各界 多管齐下推出各类举措。一是减免费用。为了激发 老年人的参与热情, 相关机构出台了减免政策, 免 费为老年人提供培训课程。二是嘉奖参与者。利用 各种机制, 对使用信息技术的积极者和踊跃者进行 嘉奖。这些举措极大地激发了老年人利用信息新媒 介参与社会生活、学习和交流的热情。三是鼓励互 助。相关培训结构鼓励老年人在学习信息技术的过 程中互帮互助, 互相交流分享学习心得, 历届优秀 者还被邀请回来担任机构的导师或者助教, 极大促 进了老年人们的数字共融。

\section{4. 多方参与, 开发老年数字产品和服务}

为了让老年人更好的数字融入，近几年香港呼吁 多方参与开发老年数字产品和服务。一是会展带动。 从 2017 年起，香港每年举办一届 “乐龄科技博览暨 高峰会” 以展示本土与世界各地相关产品及理念, 提升社会各界对老年科技应用认知, 进而促进交流 与合作 ${ }^{[15]}$ 。二是基金推动。从 2018 年起, 香港特区 政府设立了十亿港元的 “乐龄及康复科创应用基 金” ，用于资助各类涉老活动和科技服务，以提升 老年服务效能和质量, 减轻相关人员的负担 ${ }^{[16]}$ 。

上述各类举措极大地激发了老年人利用信息新 媒介参与社会生活、学习和交流的热情, 让老年人 拥有更多机会接触并融入数字化生活, 从而有利于 实现数字鸿沟的跨越, 实践证明收效良好。

\section{4. 香港老年群体数字融入的经验启示}

综上所述, 香港从深层次、多维度和跨领域来推 动老年人的数字融入和弥合老年数字鸿沟, 效果十 分显著。尽管这些举措和经验根植于香港这一特定 环境, 但是对于中国内地老年人的数字融入, 香港 的有益经验仍值得我们借鉴。

\section{1. 加强政府引领全面推动}

从香港的经验看, 政府引领全面推动对老年人数 字融入至关重要。比如政府通过设立基金、推行项 目和搭建平台等, 带动社会各界和市场投身其中, 促使各方形成共识、合力和合作共同推进老年人数 字融入。当前, 中国内地的老年事业也由政府主导, 但是对老年人数字融入的推进相对滞后, 联动机制 尚未建立健全，政府主导的作用和优势发挥有待充 分。另外, 各省市情况不一, 城乡和区域之间信息 化呈现不均衡发展, 因而老年人数字融入进程具有 多样化特点。所以, 在推进老年人数字融入的过程 中, 既要考虑顶层设计, 也要在实践中因地制宜引 导和整合社会力量, 以形成纵横贯通的联动机制, 在政策、项目和经费等方面创造条件, 为老年人数 字融入 “开路架桥”。

\section{2. 维护老年人“数字权利”}

在信息化社会, 数字融入是老年人的一项基本权 利。我国香港从人权的全方位角度出发推进老年人 数字融入, 无论是对健康老人还是失能老人, 居家 老人还是养老机构的老人都给予了充分关注, 老年 人 “数字权利” 得到充分的保障, 因而享受到越来 越多的数字红利。在我国内地, 老年人的数字融入 较低, 而且老年人的情况更加复杂, 老年人的 “数 字权利” 未得到应有保障, 他们仍是 “沉默的大多 数”。因此, 需要政府出台相关法律给予保护, 弥 补老年人在资源禀赋和社会建构上的弱势。此外, 社会各界也要充分挖掘老年人数字化生活的多元需 求, 不仅要考虑健康老年人的需求也要重视失能老 年人的需求, 让更多老年人能够享受数字红利, 进 而更加独立自主、有尊严和有质量地生活。

\section{3. 不断提升老年人数字素养}

提升数字素养是跨越数字鸿沟的关键步骤, 培训 是提升老年人数字素养的重要渠道。多年来, 香港 一直致力于老年人数字素养的提升, 无论是政府部 门还是社会机构都在持续创造和扩大老年人数字素 养培训机会, 在培训方式、培训对象、培训内容、 培训机构和经费来源等方面具有鲜明的特色。相比 之下, 我国内地其他省市在这方面尚未充分重视, 亟需加强。因此, 相关机构需要多方联动、形成教 育合力, 创新教学形式, 扩大教育覆盖面, 推动教 育内容精细化、多样化和时代性, 不断提升老年人 的数字素养。

\section{4. 促进数字长幼共融}

“长幼共融”理念贯彻于香港推进老年人数字 融入过程始终。无论是在家庭里, 还是培训机构, 长幼共融都被倡导和推崇, 这不仅有利于老年人数 字素养的提升, 而且进一步拉近了长幼间的距离, 
大大增进了交流，促进了代际和谐。相关研究表明， 代际反哺是跨越数字鸿沟的可行办法 ${ }^{[17][18]}$ 。因此, 内地在推进老年人数字融入的过程中，也应注重提 倡 “长幼共融”，这不仅有助于缩小老年数字鸿沟 以帮助老年人适应数字化生活, 更有利于增进代际 的相互理解，拉近老年人与家人、社会之间的距离, 从而有助于降低孤独感和提高幸福感。

\section{REFERENCES}

[1] NATIONS, U.World Population Prospects 2019: Highlights. Retrieved March 25, 2021, from https:// population. un. org/ wpp/Publications/Files/ WPP2019_Highlights. Pdf, 2019.

[2] Qiao Ailing, Zhang Weiyuan,and Yang Ping." A survey report on the current situation of lifelong learning among older people in the Internet era." e-Education Research 40.07 (2019):121-128. doi: 10. 13811/j. cnki.eer. 2019.07.015.

[3] Swindell, R. , \& Mayhew, C. Educating the isolated ageing: Improving the quality of life of the housebound elderly through educational teleconferencing. International Journal of Lifelong Education, 1996,15(2), 85-93.

[4] Huang, Dan-Yu, Qiu, Zi-Qing. A framework for assessing media and information literacy of older adults in the digital environment[J/OL].Library Tribune:1-13[2021-04-06].http://kns.cnki.net/kcm s/detail/44.1306.g2.20210330.1333.004.html.

[5] Yifan Yang, AND Junhao Pan." Digital inclusion dilemmas of elderly groups and response paths." News and Writing. 03(2021):22-29. doi: CNKI:SUN:XWXZ.0.2021-03-006.

[6] Friemel, T. N. The digital divide has grown old: Determinants of a digital divide among seniors. New Media \& Society, 2016,18(2), 313-331.

[7] Dutton, W. H., Blank, G., \& Groselj, D. Cultures on the Internet. InterMEDIA, Winter, $15,2014$.

[8] Yang, J. Y. "Digital Opportunities for Digital Refugees - Policy Priorities for Creating an Inclusive Information Society." Youth Journalist .24 (2013): 38-39. doi: 10. 15997/ j.cnki.qnjz.2013.24.018.

[9] Liu, Shu." A study on digital inclusion pathways for older adults in Hong Kong, China, from the perspective of active aging." Distance Education in 03(2021):67-75.doi:10.13541/j.cnki.chinade.2021. 03.008 .
[10] Huang, Chenxi." The current situation, challenges and countermeasures of the digital divide among the elderly." People's Tribune 29 (2020):126-128. doi: CNKI: SUN: RMLT.0.2020-29-047.

[11] Huang, Chenxi." The current situation, challenges and countermeasures of the digital divide among the elderly." People's Tribune .29 (2020):126-128. doi: CNKI: SUN: RMLT.0.2020-29-047.

[12] Yifan Yang, AND Junhao Pan." Digital inclusion dilemmas of elderly groups and response paths." News and Writing. 03(2021):22-29. doi: CNKI:SUN:XWXZ.0.2021-03-006.

[13] Office of the Government Chief Information Officer, Hong Kong Special Administrative Region Government. Digital Inclusion Mobility Application [EB/OL]. [2021-03-25]. https://www.ogcio.gov.hk/tc/our_work/community /past_di_initiatives/develop_mobile_apps/, 2018.

[14] Office of the Government Chief Information Officer, Hong Kong Special Administrative Region Government. Digital Plan for the Elderly [EB/OL].

[2021-03-25]. https://www.ogcio.gov.hk/tc/our_work/community /ict_programmes_for_elderly/, 2019.

[15] Hong Kong Council of Social Service. Exhibition Overview [EB/OL]. [2021-03-25]. https:// gies2017. hkcss. org. hk/ cn/about-gies.html\#background, 2017.

[16] Labour and Welfare Bureau of the Hong Kong Special Administrative Region Government. The development of ageing technology [EB/ OL]. [2021-03-25].

https://www.lwb.gov.hk/sc/blog/post_17112019. html, 2019.

[17] Zhou, Yuqiong." Digital generation gap and cultural feedbacks: a quantitative examination of the "silent revolution" within families." Modern Communication (Journal of Communication University of China) 36.02 (2014): 117-123. doi: CNKI: SUN:XDCB.0.2014-02-025.

[18] Zhu Xiuling." Adolescents' cell phone use, digital generation gap and cultural feedbacks--based on an empirical analysis of middle school students' families in Zhangzhou City, Fujian Province." Journalism and Mass Communication Monthly.11 (2015):47-53. doi: $10 . \quad 15897 / j$. cnki.cn51-1046/g2.2015.11.011. 